\title{
The Way to Evenly Maintain Water Temperature
}

\author{
$\mathrm{Xu} \mathrm{Liu}{ }^{1}$, Siwen Jiang ${ }^{2}$, Chenguang Zhang ${ }^{3}$ and Peizhi Wang ${ }^{3}$ \\ ${ }^{1}$ Room 501, No. 2, $12^{\text {th }}$ Building, Bangxing Residential, Jiangbei District, Chongqing, China \\ ${ }^{2}$ Chongqing Jiaotong University, Nanan District, Chongqing, China \\ ${ }^{3} 10^{\text {th }}$ Dormitory, Campus A, Chongqing University, Shapingba District, Chongqing, China
}

\begin{abstract}
In order to determine the best strategy for a person to get venly maintained temperature throughout the bath water in a simple water vessel without wasting too much water, we employ methods in Pyrology to build models and simulate and analyze the water temperature distribution in the bathtub under. By comparing results, we determine a practical strategy under the most common conditions.
\end{abstract}

Keywords-temperature distribution; heat dissipation; heat transfer; Pyrology; Fourier's Law

\section{INTRODUCTION}

Everyone more or less takes a bath in their daily life. However, the temperature can hardly be evenly maintained throughout the bathtub. In order to find out a way that people can adopt to better enjoy their bath, we investigate the water temperature distribution in the most common kind of bathtub, that is, a simple water vessel made of Acrylic. We determine the best strategy to keep the temperature even throughout the tub and theoretically equal to the initial temperature. In section 2 and 3 we provide the assumptions, symbols and definitions which will be useful in the following parts. In section 4 , we build up two models, that is, the heat transfer and heat dissipation model. Then, we combine and analyze our models. We come up with the best approach to get evenly maintained water temperature throughout the bathtub.

\section{ASSUMPTIONS AND JUSTIFICATION}

- The person in the tub can only control the place where he sits, the water temperature and flow rate of the faucet and the location of the faucet water outlet port. The location of the faucet water outlet port can be easily changed by moving the tub or the water outlet port of the faucet. And the water temperature and flow rate of the faucet can be adjusted by the regulator of the faucet.

- Overflow water distributes evenly over the brim of the bathtub. We assume that the bathtub is a simple water container and excess water escapes through an overflow drain. Therefore we can also assume that excess water escape evenly over the brim of the bathtub.

The temperature of the air and the ground under the tub do not change during the bath. On one hand, the time needed for bath is relatively short, which is no more than one hour in most cases. On the other hand, the capacity for the ground and air to absorb heat is relatively large in light of its massive volume.

\section{SYMBOLS AND DEFINITIONS}

\begin{tabular}{|c|c|c|}
\hline Sym & Definition & Units \\
\hline$T_{b}$ & Average temperature of human skin & ${ }^{\circ} \mathrm{C}$ \\
\hline$\phi_{i}$ & Heat flux & $W$ \\
\hline$A_{i}$ & Surface area & $n \approx E$ \\
\hline$t_{w}$ & Water surface temperature & ${ }^{\circ} \mathrm{C}$ \\
\hline$t_{a}$ & Air temperature & ${ }^{\circ} \mathrm{C}$ \\
\hline$t_{1}$ & Land surface temperature & ${ }^{\circ} \mathrm{C}$ \\
\hline $\boldsymbol{u}$ & Latent heat of water vaporization & $\operatorname{kak} \xi K g$ \\
\hline$v_{a}$ & Air flow rate around the bathtub & $n \approx \varepsilon_{Z}$ \\
\hline$P_{a}$ & $\begin{array}{l}\text { Saturated vapor pressure } \\
\text { (at the average water temperature in the bathtub) }\end{array}$ & $m w \pi R$ \\
\hline$P_{q}$ & Air vapor pressure & $n m w R_{g}$ \\
\hline$\varepsilon$ & Blackness coefficient & \\
\hline $\boldsymbol{\sigma}$ & Stefan-Boltzmann constant & \\
\hline$t$ & Time & 5 \\
\hline$\rho$ & Water density & $K g \notin n n^{2}$ \\
\hline C & Specific heat capacity of water & $f E\left(K g={ }^{\circ} \mathrm{C}\right)$ \\
\hline$A_{b}$ & The person's body surface area & $m^{2}$ \\
\hline$\mu$ & Multiplying factor for the side of the tub & \\
\hline$d$ & The diameter of the faucet & $m m$ \\
\hline$\lambda$ & Thermal conductivity coefficient for Acrylic & $M E \xi(n+K)$ \\
\hline 9 & Ideal heat source temperature & ${ }^{\circ} \mathrm{C}$ \\
\hline $\boldsymbol{H}$ & The height of the tub & $m m$ \\
\hline
\end{tabular}

FIGURE I. .

\section{Two BASIC Models}

A. Information and Data for Our Basic Models

We assumed that the person can only control the location of water outlet port of the faucet and the water temperature and flow rate of the faucet. Therefore, to establish our basic models, we provide necessary information and reasonable values for other parameters which can also influence our result.

Values for Parameters, Necessary Information and Extra Assumptions:

1 :The shape of the bathtub is a cylinder. Cylinder is one of the most common shapes for water vessels. $2:^{T_{b}}=34.93^{\circ} \mathrm{C}$. [1]

3: $d=16 \mathrm{~mm}$. We choose a common value of the diameter of the faucet. 4: $H=400 \mathrm{~mm}$. This is a common value. 5: $r=750 \mathrm{~mm}$. According to the volume and the value of height, we can obtain this value. $6: \mathrm{q}=45^{\circ} \mathrm{C} .7:^{T_{a}}=20^{\circ} \mathrm{C}$. This is a reasonable temperature for air. 8: $\varepsilon=0.96$ [1]. 
9: $u=581.4 \mathrm{kal} / \mathrm{Kg}$ 10: ${ }^{v_{a}}=0$. We neglect the air flow. 11: $P_{b}=49.9 \mathrm{~mm} \mathrm{Hg}$ 12: $P_{q}=15.6 \mathrm{mmHg}$ 13: $P=760 \mathrm{mmHg}$

14: $h_{\mathrm{wt}}=500 \mathrm{~kJ} / \mathrm{Kg}$ 15: $h_{t a}=10 \mathrm{~kJ} / \mathrm{Kg} \quad$ 16: $h_{t l}=5 \mathrm{~kJ} / \mathrm{Kg}$

17: $h_{w a}=8 \mathrm{~kJ} / \mathrm{Kg}$ 18: $h_{w b}=500 \mathrm{~kJ} / \mathrm{Kg}$ 19: $\lambda_{a}=0.19$ [2]

20: $\bar{t}=38^{\circ} \mathrm{C} 21:{ }^{t}=20^{\circ} \mathrm{C}$ 22: $d=16 \mathrm{~mm}$

\section{B. The Heat Transfer Model}

1) Obtain the heat conduction differential equation. Because the density of hot water is lower than cold water, we should place the water outlet port of the faucet as close as possible to the bottom of the bathtub and the port of the faucet should face the center of the bottom to get more uniformed temperature distribution. Moreover, to guarantee that the person can move freely in the tub, water outlet port of the faucet should be placed as close as possible to the sidewall of the tub. Therefore, we conclude that the person should place the faucet at the intersection of the sidewall and the bottom of the tub and make water outlet port face the center of the bottom. In order to analyze the distribution of the temperature in the tub, we need to regard the water outlet port of the faucet as an ideal heat source.

According to Fourier's Law, we know that in order to obtain the heat flow of an object, we must first know the temperature field of it. To obtain the temperature field, we need to establish the equation which describes the general laws of temperature field, namely, the Heat Conduction Differential Equation[3].

The Heat Conduction Differential Equation in time and space is as follows:

$$
\rho c \frac{\partial T}{\partial t}=\frac{\partial}{\partial x}\left(\lambda \frac{\partial T}{\partial x}\right)+\frac{\partial}{\partial y}\left(\lambda \frac{\partial T}{\partial y}\right)+\frac{\partial}{\partial z}\left(\lambda \frac{\partial T}{\partial z}\right)+Q
$$

According to the shape and size of the tub, the temperature of water and air, we can obtain the initial conditions, which are as follows:

The geometry boundary conditions:

The three dimensional coordinate system we construct and the geometry boundary conditions are illustrated in Figure 1, we choose the center of the tub bottom as the origin of coordinates.

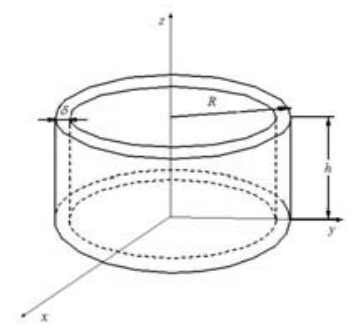

FIGURE II. THE GEOMETRY BOUNDARY CONDITIONS

The physical boundary conditions

$$
\lambda_{w}=63.06 \mathrm{~W} /(m \cdot K) .
$$

$$
T_{0}=f(x, y, z, 0)=38^{\circ} \mathrm{C} .
$$

Neumann boundary conditions:

The boundary condition for the front and the rear surfaces is

$-\lambda \frac{\partial T}{\partial x}=h\left(f(x, y, z, t)-t_{a}\right)$

The boundary condition for the left and the right surfaces is

$-\lambda \frac{\partial T}{\partial y}=h\left(f(x, y, z, t)-t_{a}\right)$

The boundary condition for the left and the right surfaces is

$-\lambda \frac{\partial T}{\partial y}=h\left(f(x, y, z, t)-t_{a}\right)$.

The boundary condition for upper surface is

$-\lambda \frac{\partial T}{\partial z}=h\left(f(x, y, z, t)-t_{a}\right)$

The boundary condition for lower surface is

$-\lambda \frac{\partial T}{\partial z}=h\left(f(x, y, z, t)-t_{l}\right)$

2) Reduce the dimension of equations. Combine the above seven equations, we can obtain second-order linear nonhomogeneous partial differential equations set with four unknowns. Because of the limitation of time and knowledge, we cannot work out the general solution to those equations. In order to continue our analysis, we reduce the dimension of the seven equations. Moreover, analyzing $x-y$ plane is more meaningful for us because we can get the heat distribution in the tub in a given depth. In light of this, we decide to assume that $\mathrm{z}$ is constant. This means that the value of $\partial T / \partial \mathrm{z}$ is zero. We can obtain a two-dimensional equations, which are as follows:

$\left\{\begin{array}{l}\rho c \frac{\partial T}{\partial t}=\frac{\partial}{\partial x}\left(\lambda \frac{\partial T}{\partial x}\right)+\frac{\partial}{\partial y}\left(\lambda \frac{\partial T}{\partial y}\right)+Q \\ T_{0}=f(x, y, z, 0)=38^{\circ} \mathrm{C} \\ -\lambda \frac{\partial T}{\partial x}=h\left(f(x, y, z, t)-t_{a}\right) \\ -\lambda \frac{\partial T}{\partial y}=h\left(f(x, y, z, t)-t_{a}\right)\end{array}\right.$

Based on (8), we can do simulation in Matlab. We can obtain temperature distribution in the bathtub at certain depth[4].

3) Simulation and Analysis

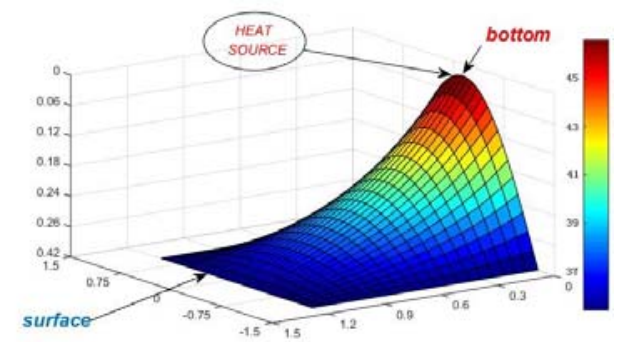

FIGURE III. THE IMAGE OF NUMERICAL VALUES IN TEMPERATURE 


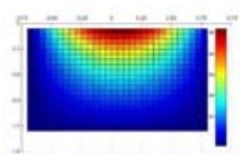

Figure $4 z=0$

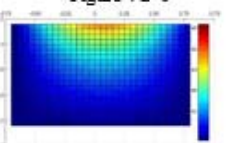

Figure $7 z=15$

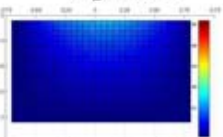

Figure $10 z=30$

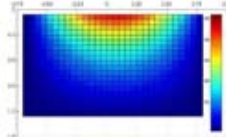

Figure $5 z=5$

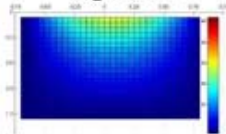

Figure $8 z=20$

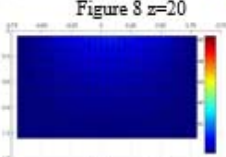

Figure $11 \mathrm{z}=35$

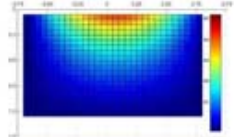

Figure $6 z=10$

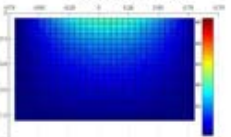

Figure $9 z=25$

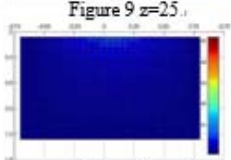

Figure $12 z=40$.
FIGURE IV.

Nine figures show the temperature distribution on the $x-y$ plane in different depth. In other words, values of the $\mathrm{z}$ coordinate are different in those figures. And the exact values of the $\mathrm{z}$ coordinate are presented under each figure. (Our threedimensional coordinate system was shown in Figure2)

From the above pictures, we can draw the following conclusions:

The average temperature of the cross section of the tub decreases as the value of the $\mathrm{z}$ coordinate increases.

The distribution of the temperature become more uniform the $\mathrm{z}$ coordinate increases.

The temperature of sidewall where the water outlet port lies is higher than that of the opposite sidewall. This is the reason why we place the person opposite to the sidewall where water outlet port is.

The Figure11 shows that the water temperature distribution is approximately even and the temperature is around $36^{\circ} \mathrm{C}$. Therefore we decide the average temperature of overflow water to be $36^{\circ} \mathrm{C}$.

In more than two thirds of the space in the tub, except those areas very close to the outlet port of the faucet, the range of temperature is no more than $3^{\circ} \mathrm{C}$, which will have little influence on the person.

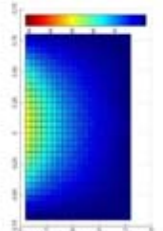

Figure $13 \mathrm{t}=12$

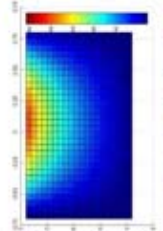

Figure $14 \mathrm{t}=24$

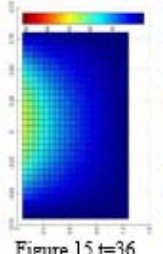

FIGURE V.

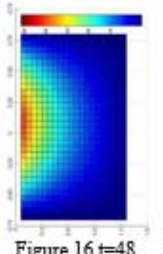

Figure $16 t=48$ Figure $17 t=60$
The five pictures above illustrates how the temperature distribution on the middle cross section of the tub $(\mathrm{z}=20)$ changes over time. From the comparison and analysis of those pictures, we can come up with the following results:

The temperature distribution is fluctuate periodically, and the time cycle is about 24 seconds.
The fluctuation near the water outlet port of the faucet is more intense, which means those areas are not suitable for the person to stay. This is another reason for us to place the person at the opposite side.

Overall, the temperature fluctuation in any place of the middle cross section is no more than $2^{\circ} \mathrm{C}$, which will have little influence on the person.

\section{The Heat Dissipation Model}

1) Establish model for heat dissipation. According to the law of conservation of energy, to maintain the temperature of the water in the bathtub, the dissipation of heat in the tub water must equals the input heat. And the input heat comes from the input water. Since we have determined the temperature of the water, we can determine the flow rate of water once we obtain the heat dissipation rate. Therefore, in order to figure out the water flow rate of the faucet, we need to calculate the total heat loss. We take following six main ways the heat dissipate into consideration and we calculate their respective heat loss.[5]

The heat escapes through the side wall to the air:

$$
\phi_{1}=\frac{t_{w}-t_{a}}{\frac{1}{A_{1} h_{w t}}+\frac{\delta}{\lambda A_{1}}+\frac{1}{A_{1} h_{t a}} .}
$$

The heat escapes through the side wall into the ground:

$$
\phi_{2}=\frac{t_{w}-t_{l}}{\frac{1}{A_{2} h_{w t}}+\frac{\delta}{\lambda A_{2}}+\frac{1}{A_{2} h_{t l}} .}
$$

The heat escapes through the convection on the top surface:

$$
\phi_{3}=\frac{\frac{t_{w}-t_{a}}{1}}{\frac{1}{A_{3} h_{w a}}}
$$

The evaporation of water:

$$
\phi_{4}=1.163 \times u \times\left(0.174 v_{a}+0.0229\right) \times\left(P_{b}-P_{q}\right) \times A_{3} \times 760 / P .
$$

The heat dissipate through radiation between the top surface and the walls of the room:

$$
\phi_{5}=A_{3} \cdot \varepsilon \cdot \sigma \times\left(t_{w}-t_{a}\right) .
$$

The heat escapes into the person in the bathtub:

$$
\phi_{6}=\frac{\frac{t_{w}-t_{b}}{1}}{\frac{1}{A_{b} h_{w b}} .}
$$

2) Solutions and Analysis

First, we plug the values of parameters into the above six equations, then we can obtain the results for those equations:[6] (unit: $W$ )

$$
\begin{array}{cccr}
\phi_{1}=163.62 & \phi_{2}=100.98 & \phi_{3}=254.88 \\
\phi_{4}=940.06 & \phi_{5}=191.52 & \phi_{6}=3330 & \phi_{\text {sum }}=4981.06
\end{array}
$$

According to the basic knowledge in Physics, we know that

$$
\frac{C m \cdot \Delta T}{\Delta t}=\phi
$$

Because that 


$$
\begin{aligned}
m & =\rho \cdot \Delta V . \\
\Delta V & =\left(V-V_{b}\right) .
\end{aligned}
$$

Therefore, we can obtain that

$$
\frac{C \cdot \rho \cdot \Delta V \cdot \Delta T}{\Delta t}=\phi
$$

Transform the equation and we can obtain that

$$
\Delta T=\frac{\phi \cdot \Delta t}{C \cdot \rho \cdot \Delta V} .
$$

Based on the above equation, we can draw the Figure17

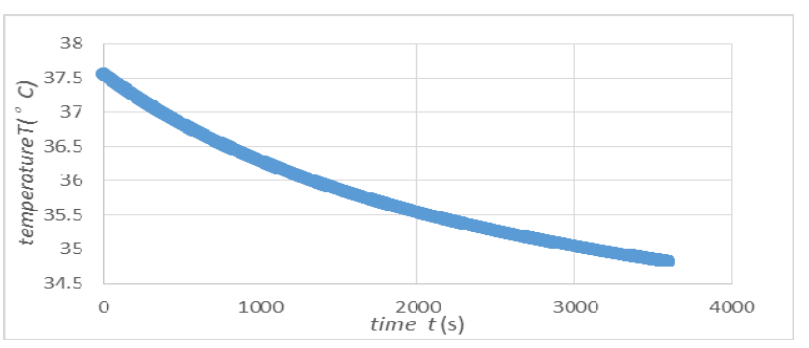

FIGURE VI. THE CURVE THAT DESCRIBE HOW THE TEMPERATURE CHANGE WITH THE TIME

From Figure 17 we can conclude that the temperature drops noticeably overtime. After one hour, the temperature decreases for about $3{ }^{\circ} \mathrm{C}$

From basic Physics knowledge, we know that:

$$
m / \Delta t=\rho \cdot q
$$

Plug (10) into (9), we can come up with the following equation:

$$
C \cdot \rho \cdot q \cdot \Delta T=\phi
$$

Transform the equation and we can obtain that:

$$
q=\frac{\phi}{C \rho\left(T-t_{w}\right)}
$$
that

Plug the value $\phi=\phi_{\text {sum }}=4981.06$ into (11), we can obtain

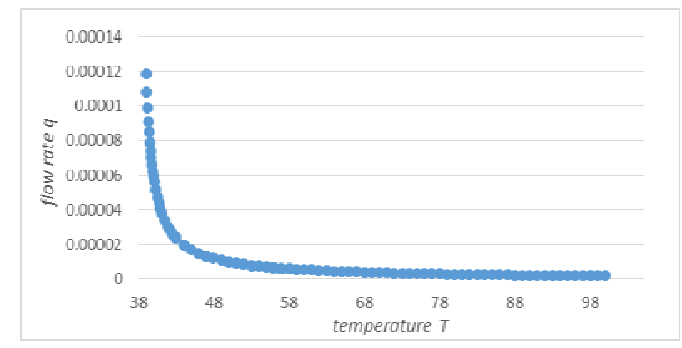

FIGURE VII. THE CURVE THAT DESCRIBES HOW THE TEMPERATURE CHANGES WITH THE FLOW RATE

The temperature of the human body skin can feel is $20^{\circ} \mathrm{C}$. $47^{\circ} \mathrm{C}$ between, when the temperature at about $35^{\circ} \mathrm{C}$, the human body skin can feel sensation of warmth; when temperature reach $45^{\circ} \mathrm{C}$, even feel hot; When the temperature reach $47^{\circ} \mathrm{C}$, feeling painful; At more than $50^{\circ} \mathrm{C}$ temperature, people will be scald form blister; If $60^{\circ} \mathrm{C}$ hot water in contact with human skin in 1 minute, it can cause III degree burns.
In conclusion, we choose the temperature of the hot water is $45^{\circ} \mathrm{C}$. Then we can figure out that:

Because we know that:

$$
q=1.694 \times 10^{-4} \mathrm{~m}^{3} / \mathrm{s}=10 \mathrm{~L} / \mathrm{min} .
$$

$$
d=16 \mathrm{~mm} \text {. }
$$

Therefore we can know the area of the outlet port of the faucet that:

$$
A_{d}=\frac{\pi d^{2}}{4}=2 \times 10^{-4} \mathrm{~m}^{3} .
$$

Therefore we can obtain that

$$
v_{d}=0.847 \mathrm{~m} / \mathrm{s} .
$$

From the analysis above, we can draw the following conclusions:

If the person do not add hot water, the average temperature of the water in the tub will drop $6.73^{\circ} \mathrm{C}$ per hour. This manifest that our model correspond with the conditions of the problem that the water can be noticeably colder after a while.

From our models, we can know that In order to maintain the same temperature over time, the person should add a trickle of water whose flow rate $v_{d}$ is around. This a reasonable flow rate and the most common faucet can provide this flow rate.

\section{CONCLUSIONS FOR THE TwO BASIC MODELS}

From the above discussion, we can come up with some general conclusions to our two basic models, which are as follows:

The temperature distribution is even throughout most space in the bathtub except those very close to the hot water inlet port. In other words, the temperature distribution became more uniform as the distance to the port increases.

The temperature distribution fluctuates periodically over time, however, the fluctuation is not so conspicuous and it will not have much influence on the person in the bathtub.

If there is hot water coming in, the average temperature of the bathtub will be $38^{\circ} \mathrm{C}$.

If the person wants to maintain the temperature in the bathtub, he need to keep the water flow rate to be $0.847 \mathrm{~m} / \mathrm{s}$

\section{REFERENCE}

[1] Chen li. The heat analysis of the body surface temperature distribution of ordinary teenager.

[2] Thermal conductivity http://sh.to8to.com/company/z4982.html

[3] Li Chunfeng. Application of the mathematic modeling in heat transfer. 2005.8

[4] A Numerical Method Based On Finite Element for Two Dimensional Initial-Boundary Value Heat Conduction Equation -Numerical Realize With MATLAB 2011.6 Civil Aviation University of China

[5] Zhang Xuexue basic Pyrology. 2006.Higher Education Press.

[6] Zuber N. On the stability of boiling heat transfer[J], Trans ASME,1958.

[7] Mathematical Contest In Modeling. COMAP Inc. 2016 